\title{
A STRUCTURE THEOREM FOR TOPOLOGICAL LATTICES
}

\author{
by L. W. ANDERSON and L. E. WARD, JR \\ (Received 4 January, 1960)
}

In the study of connected partially ordered spaces a problem of fundamental interest is to determine sufficient conditions to ensure the existence of chains (i.e., simply ordered subsets) which are connected. Recently [5] R. J. Koch proved that, if $X$ is a compact Hausdorff space with continuous partial order (i.e., the partial order has a closed graph), if $L(x)=\{y: y \leqq x\}$ is connected for each $x \in X$, and if $X$ has a zero (i.e., an element 0 such that $0 \leqq x$ for all $x \in X$ ), then each element of $X$ lies in a connected chain containing zero. It is easy to find simple examples which show that this result is false if $X$ is assumed only to be locally compact. However, if it is assumed that the partial order is that of a topological lattice then the existence of such chains can be shown by elementary methods. This solves a problem which was proposed in [3].

Recall that a topological semilattice can be defined to be a partially ordered Hausdorff space $(S, \leqq)$ such that the operation $x \wedge y=$ g.l.b. $(x, y)$ is defined and continuous on $S \times S$. If, in addition, the operation $x \vee y=$ l.u.b. $(x, y)$ is defined and continuous, then $(S, \leqq)$ is a topological lattice. It is known $[1,4]$ that the partial order is continuous in a topological semilattice. Moreover, if $S$ is connected then so is $x \wedge S=L(x)$ for each $x \in S$.

Let $a$ and $b$ be elements of a partially ordered space with $a \leqq b$. We say that $a$ is chained to $b$ provided that the space contains a connected chain $C$ such that $a=\inf C$ and $b=\sup C$. In addition, $C$ is said to be a chain from $a$ to $b$. It follows from [6] that if the space is locally compact then such a chain is compact.

Finally, we recall that a subset $C$ of a partially ordered set $S$ is convex if, whenever $x<y$ and $y<z$ with $x$ and $z$ elements of $C$ and $y \in S$, it follows that $y \in C$. A partially ordered space is locally convex provided that the topology possesses a base consisting of convex sets. A subset $K$ of a partially ordered set is order-dense if, whenever $a$ and $b$ are elements of $K$ and $a<b$, there exists an element $c$ of $K$ such that $a<c$ and $c<b$.

Lemma. Let $S$ be a connected locally compact semilattice, let $U$ be an open subset of $S$, and let $x \in U$. If $x$ has arbitrarily small closed order-dense neighbourhoods, then there exists an open set $V$, with $x \in V \subset U$, such that if $y$ and $z$ are elements of $V$ then $y \wedge z$ is chained to $z$.

Proof. Let $W$ be an open set such that $x \in W \subset U$ and $\bar{W}$ is order-dense and compact. Since $\wedge$ is continuous there exists an open set $V$ such that

$$
x \in V \subset V \wedge V \subset W
$$

If $y$ and $z$ are elements of $V$ then $y \wedge z \in W$. Let $C$ be a chain in $\bar{W}$ which is maximal with respect to containing $y \wedge z$ and $z$. Since $\bar{W}$ is a compact order-dense partially ordered space, each of its maximal chains is compact [6, Lemma 4] and order-dense, and hence $C$ is connected [6, Theorem 4]. The set $C \cap\{p: y \wedge z \leqq p \leqq z\}$ is clearly a connected chain from $y \wedge z$ to $z$. 
THEOREM 1. Let $S$ be a connected locally compact semilatice with zero and suppose that each element of $S$ has arbitrarily small closed order-dense neighbourhoods. Then zero is chained to each $x \in S$.

Proof. Let $P$ denote the set of all $x \in S$ such that 0 (zero) is chained to $x$. Obviously $0 \in P$ so that it is sufficient to prove that $P$ is open and closed. Let $x \in P$ and let $U$ and $V$ be chosen as in the lemma. If $y \in V$, then there is a connected chain $C(x \wedge y, y)$ from $x \wedge y$ to $y$. If $C$ is a connected chain from 0 to $x$ then $(C \wedge y) \cup C(x \wedge y, y)$ is a connected chain from 0 to $y$. Hence $y \in P$ and $P$ is open.

To see that $P$ is closed let $x \in \bar{P}$ and again choose $U$ and $V$ as in the lemma. Let $y \in V \cap P$, let $C$ be a connected chain from 0 to $y$ and $C(x \wedge y, x)$ a connected chain from $x \wedge y$ to $x$. Then $(C \wedge x) \cup C(x \wedge y, x)$ is a connected chain from 0 to $x$ so that $x \in P$, i.e., $P$ is closed.

We do not know whether a connected and locally compact locally order-dense semilattice necessarily satisfies the hypothesis of Theorem 1. However, for lattices the situation is simpler.

COROLlaRY 1. If $L$ is a connected and locally compact topological lattice with zero, then zero is chained to each element of $L$.

Proof. It suffices, in view of Theorem 1, to show that each point of $L$ has arbitrarily small closed order-dense neighbourhoods. Let $x \in U$, an open set in $L$. It is known [2] that $L$ is locally convex and bence $x \in V \subset U$, where $V$ is some open convex set. Let $W$ be open and $x \in W \subset \bar{W} \subset V$; if $C(\bar{W})$ denotes the smallest convex set containing $\bar{W}$ then $C(\bar{W}) \subset C(V)=V$. From [2] $C(\bar{W})$ is closed; hence $x$ has arbitrarily small closed convex neighbourhoods. To see that $C(\bar{W})$ is order-dense, let $a$ and $b$ be elements of $C(\bar{W})$ with $a<b$; then $b \wedge(a \vee L)$ is a connected subset of $C(\bar{W})$ and hence $C(\bar{W})$ contains an element $c$ such that $a<c<b$.

COROLLARY 2. If $L$ is a connected and locally compact topological lattice and if $a \leqq b$ in $L$, then $a$ is chained to $b$ in $L$.

Proof. Apply Corollary 1 to the lattice $a \vee L$.

There exists a connected and locally compact topological semilattice with zero such that zero is not chained to each point. In the cartesian plane let

$$
\begin{aligned}
A_{-1} & =\{(1, y): 0 \leqq y \leqq 1\}, \\
A_{n} & =\left\{\left(1-2^{-n}, y\right): 0 \leqq y \leqq 1\right\} \quad(n=0,1, \ldots), \\
B & =\{(x, 0): 0 \leqq x \leqq 1\}, \\
L^{\prime} & =B \cup \bigcup_{n=-1}^{\infty}\left\{A_{n}\right\} .
\end{aligned}
$$

If $L=L^{\prime}-\{(1,0)\}$ is partially ordered by $(a, b) \leqq(c, d)$ if and only if $a \leqq c$ and $b \leqq d$, then it is easy to verify that $L$ is a connected and locally compact topological semilattice with zero. However, there is no connected chain from zero to $(1,1)$.

If the topological semi-lattice is also locally connected, then it is not known whether zero is chained to each point. However, there exists a locally compact and locally connected partially ordered space $X$ satisfying these conditions: the partial order is continuous and there exists a zero, $L(x)=\{y: y \leqq x\}$ is connected for each $x \in X$, and there is a point $p \in X$ such that zero is not chained to $p$. To see this, let $X$ be the product of the closed unit interval with itself, 
with the point $(1,0)$ deleted. Define $(a, b) \leqq(c, d)$ if and only if the following condition is satisfied: if $c<1$ then either $a=c$ and $b \leqq d$ or $a \leqq c$ and $b=0$; if $c=1$ then either $a=1$ and $b \leqq d$, or $a \leqq 1$ and $b=0$, or $a=(n-1) / n$ for some positive integer $n$ and $b \leqq d$. It is a tedious but elementary exercise to verify that this relation is a continuous partial order, that $L(x)$ is connected for each $x \in X$, and that $(0,0)$ is the zero of $X$. Moreover, there is no connected chain from $(0,0)$ to $(1,1)$.

Let $I$ denote the closed unit interval of real numbers. An arcwise connected space $X$ is said to be simply connected if, given a point $a \in X$ and a continuous function $f: I \rightarrow X$ with $f(0)=f(1)$, there is a homotopy $g: I \times I \rightarrow X$ such that $g(t, 0)=f(t), g(t, 1)=a$, and $g(0, r)=g(1, r)=a$ for each $r \in I$.

THEOREM 2. If $S$ is an arcwise connected topological semilattice with zero, then $S$ is simply connected.

Proof. Let $f: I \rightarrow S$ be continuous with $f(0)=f(1)=0$. Define $g: I \times I \rightarrow S$ by $g(t, r)=f(t) \wedge f(t-t r)$.

COROLLARY 3. If $S$ is a connected and locally compact metric topological semilattice with zero and if each element of $S$ has arbitrarily small closed order-dense neighbourhoods, then $S$ is simply connected.

Proof. By Theorem 1, zero is chained to each point of $S$. It is well-known that a compact connected metric chain is an arc (see, for example, [7, p. 30]) and hence $S$ is arcwise connected.

COROLLARY 4. If $L$ is a connected and locally compact metric topological lattice with zero, then $L$ is simply connected.

\section{REFERENCES}

1. L. W. Anderson, On the distributivity and local connectivity of plane topological lattices, Trans. Amer. Math. Soc. 91 (1959), 102-112. 720.

2. L. W. Anderson, One-dimensional topological lattices, Proc. Amer. Math. Soc. 10 (1959), $715-$

3. L. W. Anderson, On the breadth and codimension of a topological lattice, Pacific J. Math. 9 (1959), 327-333.

4. L. W. Anderson and L. E. Ward, Jr, One-dimensional topological semilattices. To appear in Illinois J. Math.

5. R. J. Koch, Arcs in partially ordered spaces, Pacific J. Math. 9 (1959), 723-728.

6. L. E. Ward, Jr, Partially ordered topological spaces, Proc. Amer. Math. Soc. 5 (1954), 144-161.

7. R. L. Wilder, Topology of manifolds (New York, 1949).

UNIVERSITY OF OREGON

U.S. Naval Ordnance Test Station 